\title{
Occult fractures of the proximal femur: imaging diagnosis and management of 82 cases in a regional trauma center
}

Bogdan Deleanu ${ }^{1,2}$, Radu Prejbeanu ${ }^{1,2}$, Eleftherios Tsiridis ${ }^{6}$, Dinu Vermesan ${ }^{1,2}$, Dan Crisan $^{1 *}$ (D) Horia Haragus ${ }^{1}$, Vlad Predescu, ${ }^{4,5}$ and Florin Birsasteanu ${ }^{2,3}$

\begin{abstract}
Background: Occult hip fractures are often difficult to identify in busy trauma units. We aimed to present our institutions experience in the diagnosis and treatment of occult fractures around the hip and to help define a clinical and radiological management algorithm.

Method: We conducted a seven-year retrospective hospital medical record analysis. The electronic database was searched for ICD-10 CM codes S72.0 and S72.1 used for proximal femoral fractures upon patient discharge. We identified 34 (4.83 \%) femoral neck fractures and 48 (4.42 \%) trochanteric fractures labeled as occult.

Results: The majority of the cases were diagnosed by primary MRI scan (57.4\%) and 12 were diagnosed by emergency CT scan (14.6\%). For the remaining cases the final diagnosis was confirmed by 72 h CT scan in 9 patients (representing $39 \%$ of the false negative cases) or by MRI in the rest of 14 patients. MRI was best at detecting incomplete pertrochanteric fracture patterns $(13.45 \%$ of total) and incomplete fractures of the greater trochanter (3.65 \% of total) respectively. It also detected the majority of Garden I femoral neck fractures (20.7\% of total). CT scanning accurately detected $100 \%$ of Garden 2 fractures (2.44 \%) and $25 \%$ (3.65 \%) of the complete pertrochanteric fractures (false negative $25 \%$ ).

Conclusion: Occult fractures should be suspected in all patients with traumatic onset of hip pain that is inconsistent with normal radiographic findings. MRI is the golden standard but not as readily available not as cheap and not quite as quick to perform as as a CT scan. The latter which in turn can provide falsely negative results in the first $24 \mathrm{~h}$. Improved imaging protocols could expedite management and improve treatment.
\end{abstract}

Keywords: Occult, Hip, Fracture, X-ray, MRI, CT scan

\section{Background}

The estimated prevalence of occult hip fractures varies between 2 and $10 \%$ of the total hip fractures [1-7].

Occult are defined, those fractures that cannot be detected by radiographic standard examination until several weeks after injury [8]. Their importance resides in their "occult" status, meaning that they are not identifiable by routine emergency X-rays. In the Emergency Room (ER) most of the times only a pelvic or hip AP view is obtained, with some services requiring a complete trauma X-Ray set

\footnotetext{
* Correspondence: crisan.dan@gmail.com

${ }^{1}$ I-st Clinic of Orthopedics and Trauma, Pius Brinzeu Emergency Clinical County Hospital, 10 I. Bulbuca Blvd, 300737 Timisoara, Romania Full list of author information is available at the end of the article
}

with lateral, inlet, outlet and Judet oblique views. The necessity of these explorations is however disputed as the presence of a concomitant pelvic fracture either radiographically visible or occult and a occult proximal femur fracture has been excluded by previous studies [9]. Radiographically unapparent fractures can be easily mislabeled as hip soft tissue trauma and treated conservatively, with no restriction on weightbearing.

Definitive diagnosis of occult fractures of the proximal femur is without exception an imaging one. MRI is the imaging method of choice, better tolerated by patients providing a faster diagnosis of occult fractures of the hip, it is therefore recommended that the MRI is performed in an emergency setting whenever it is available 
$[2,4,5,10]$. But not all services can provide emergency access to MR scanning, while CT scanning is more widespread and cheaper to perform. There is still no consensus over the use of $\mathrm{CT}$ in detecting occult hip fractures and it has been demonstrated that even modern 64 slice machines can yield false negative results [11].

We aimed to present our institutions experience in the diagnosis and treatment of occult fractures around the hip and to help defining a clinical and radiological management algorithm.

\section{Method}

We conducted a seven-year retrospective search on the hospital medical record (Hipocrate, RCS Software, Bucharest, Romania) between 2005-2012. The electronic database of a regional trauma center was searched for ICD10 codes S72.0 (intracapsular) and S72.1 (extracapsular) used for proximal femoral fractures upon patient discharge. We identified 769 femoral neck and 926 pertrochanteric fractures that were treated in our clinic during this period. Out of these, 34 (4.83\%) femoral neck fractures and 48 (4.42\%) pertrochanteric fractures were labeled as occult. The fracture distribution by fracture subtype can be seen in Fig. 1.

Emergency AP radiographs routinely investigate hip trauma with the hips in neutral rotation. These are almost always sufficient for establishing a diagnosis and a management strategy for the case. For cases with suspected occult fracture further imaging was carried out either with an MRI (General Electric Signa Horizon LX 1 Tesla) or a CT scan (Philips Brilliance MX 16 slice). No contrast was used in either method. For the cases with an inconclusive emergency $\mathrm{CT}$ and eloquent hip symptoms such as pain in the Scarpa triangle, in the greater trochanter region, on weight-bearing or during passive movement of the hip joint, an MRI scan was performed during the following $24 \mathrm{~h}$ or a second CT scan at $72 \mathrm{~h}$ for establishing the diagnosis.

Five patients were self-discharged from the ER without allowing us to establish final diagnosis. All five patients were reexamined in the next 1-3 days by a senior orthopedic surgeon due to ongoing hip symptoms and an outpatient MRI scan was performed. Most patients with hip fractures are elderly patients and the occult fractures group fitted this demographic distribution with 80 patients that were aged 60 and over, out of which 67 were aged 70 or over and only two young patients who were aged less than 60 years. The patients that decided to leave the ER were all over 75 years of age and did so upon consultations with their families. Thirty-eight patients had at least one neurological condition such as Parkinson's disease, cortical atrophy or stroke history, which ranged in severity from mild to serious. The majority of diagnosed cases were treated surgically whilst conservative management was reserved for the incomplete and totally undisplaced occult fractures according to the literature [12, 13].

Suspicion of an occult hip fracture should arise when there is a inconsistency between the trauma history and physical examination on one hand and the imaging results on the other. For example a young patient with high-energy hip trauma that has a negative X-ray but presents with a nonweightbearing painful hip should be further investigated. The patient may be able to bear weight but also present significant hip pain spontaneous and/or on palpation mobilization and this should also prompt for further imaging studies. Elderly patients with occult hip fractures almost always have sustained a trivial trauma and present non or partial weightbearing with pain in the anterior portion of the hip.

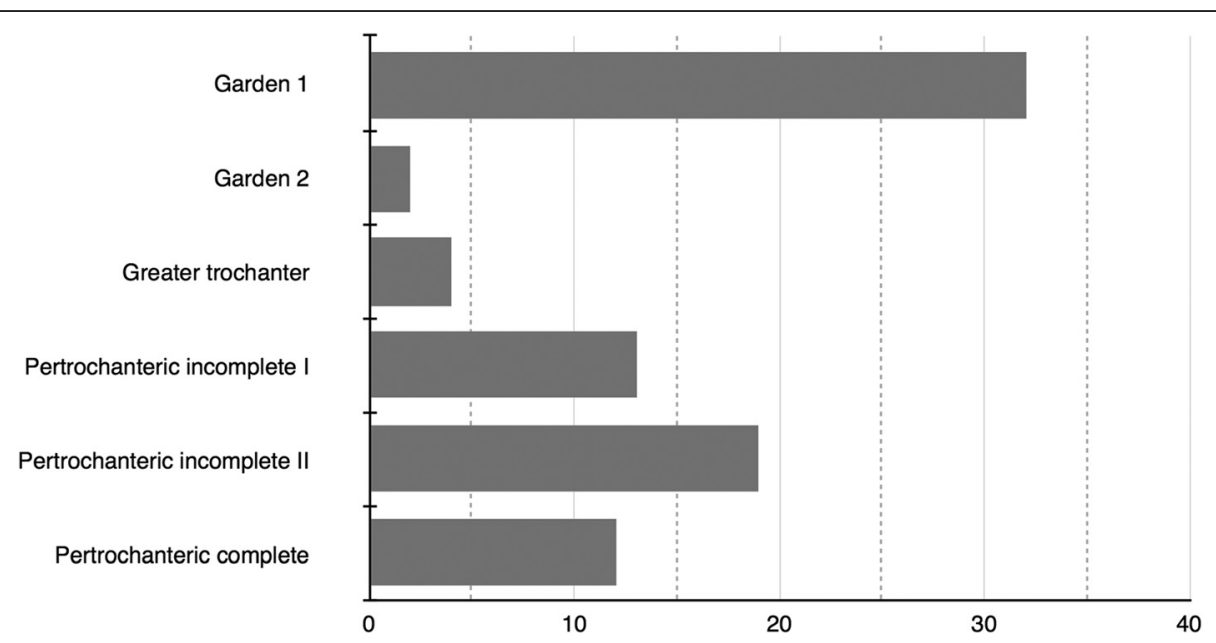

Fig. 1 Distribution of the occult fractures in fracture types. Pertrochanteric incomplete I represents $<50 \%$ of the bicortical distance fractured. Pertrochanteric incomplete II represents $>50 \%$ of the bicortical distance fractured 
All data were recorded electronically using Microsoft Office Excel spread sheets (Microsoft Corporation, Redmond, US-WA) and the statistical analysis was performed using XLStat (Addinsoft SARL New York, US-NY). Approval for this study was obtained from our Hospital's institutional review board (IRB) and informed consent was obtained from each patient.

\section{Results}

From a total of 82 identified occult fractures 47 were diagnosed by MRI (57,4 \% 95 \% CI: 27,4 \%-90,9 \%) and 12 were diagnosed by emergency CT $(14,6 \% 95 \%$ CI: $6,0 \%-21,9 \%)$. For the remaining 23 cases the final occult fracture diagnosis was confirmed by $72 \mathrm{~h}$ CT scan in 9 cases $(11 \% 95 \%$ CI: 3,7 \% - 9,5\% representing $39 \%$ of the false negative cases) or by MRI in the rest of 14 cases $(17 \% 95 \%$ CI: $3 \%-33,6 \%$, representing $61 \%$ of the false negative cases) (Fig. 2).

MRI was best $(p=0.048)$ at detecting incomplete pertrochanteric fracture patterns in $84,6 \%$ of the cases $(13,45 \%$ of total $95 \% \mathrm{CI}: 12,1 \%-20,7 \%)$ and incomplete fractures of the greater trochanter $75 \%(3,65 \%)$ and detected the majority (53 \%) of Garden I femoral neck fractures (20,7 \% of total). CT scanning accurately detected $100 \%$ of Garden 2 fractures $(2,44 \%$ of total) and $25 \%$ of the complete pertrochanteric fractures $(3,65 \%$ of total) but with more false negative results ( 23 false negative results based on acute scan; $28 \%$ of total).

For femoral neck fractures in the elderly (Fig. 3a, b and c), the treatment of choice was hip hemiarthroplasty with a bipolar head and an uncemented stem (Taperlock,
Biomet, Warsaw). Three parallel neck screws were used for 2 young patients with good bone stock. Pertrochanteric fractures (Fig. 3d, e and f) were routinely treated by intramedullary nailing (Gamma, Stryker, New Jersey) or extramedullary fixation with a sliding hip screw. Both techniques allowed immediate weight-bearing and early mobilization. Femoral neck fractures concomitant or iatrogenic were not detected in the patients undergoing nailing.

Out of the 82 cases investigated 34 were managed conservatively. Non-operative treatment consisted of bed rest initially and nonweightbearing on the affected leg no sooner than 3 weeks from the trauma. No attempt to manipulate the fracture was made since these were all nondisplaced fractures. No immobilization or skeletal traction was used. Progressive weightbearing was initiated at 6 weeks, starting with $10 \%-20 \%$ of total weight and increasing at a rate of $10 \%-15 \%$ per week under the direct supervision of a physical therapist. Radiographic evaluation was performed weekly for the first 4 weeks and monthly for the subsequent 3 months.

The relatively high rate of nonoperative treatment (34 cases, $46 \%$ of total) was the result of a number of factors namely: incomplete fractures of the greater trochanter (4 cases, $4,9 \%$ ) and incomplete pertrochanteric fractures (13 cases, 15,8\%) In those cases the risk to benefit ratio would not justify the surgical intervention and the consensus of the trauma meeting of our units was in favor of conservative management. Furthermore associated comorbidities (7 cases, $8,5 \%$ ) were another rationale to selecting conservative treatment and finally the patient choice (10 cases, $12,2 \%)$.

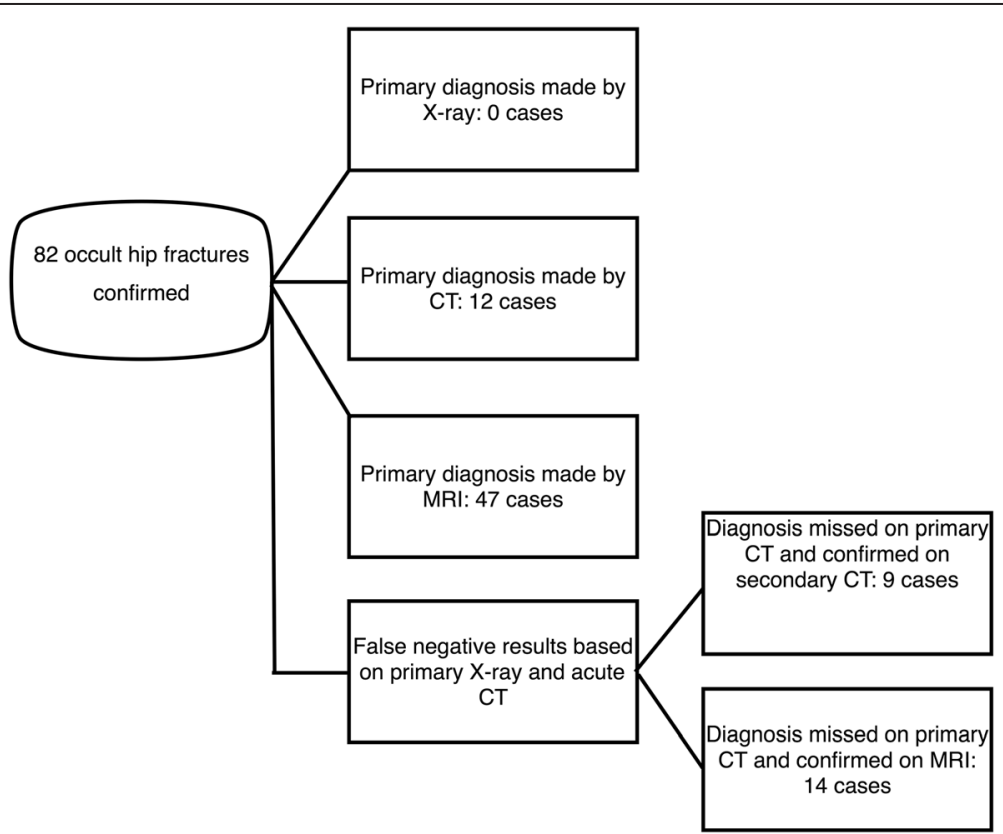

Fig. 2 Case stratification showing the diagnostic algorithm used and the number of fractures diagnosed primarily and secondarily by MRI and CT scan 


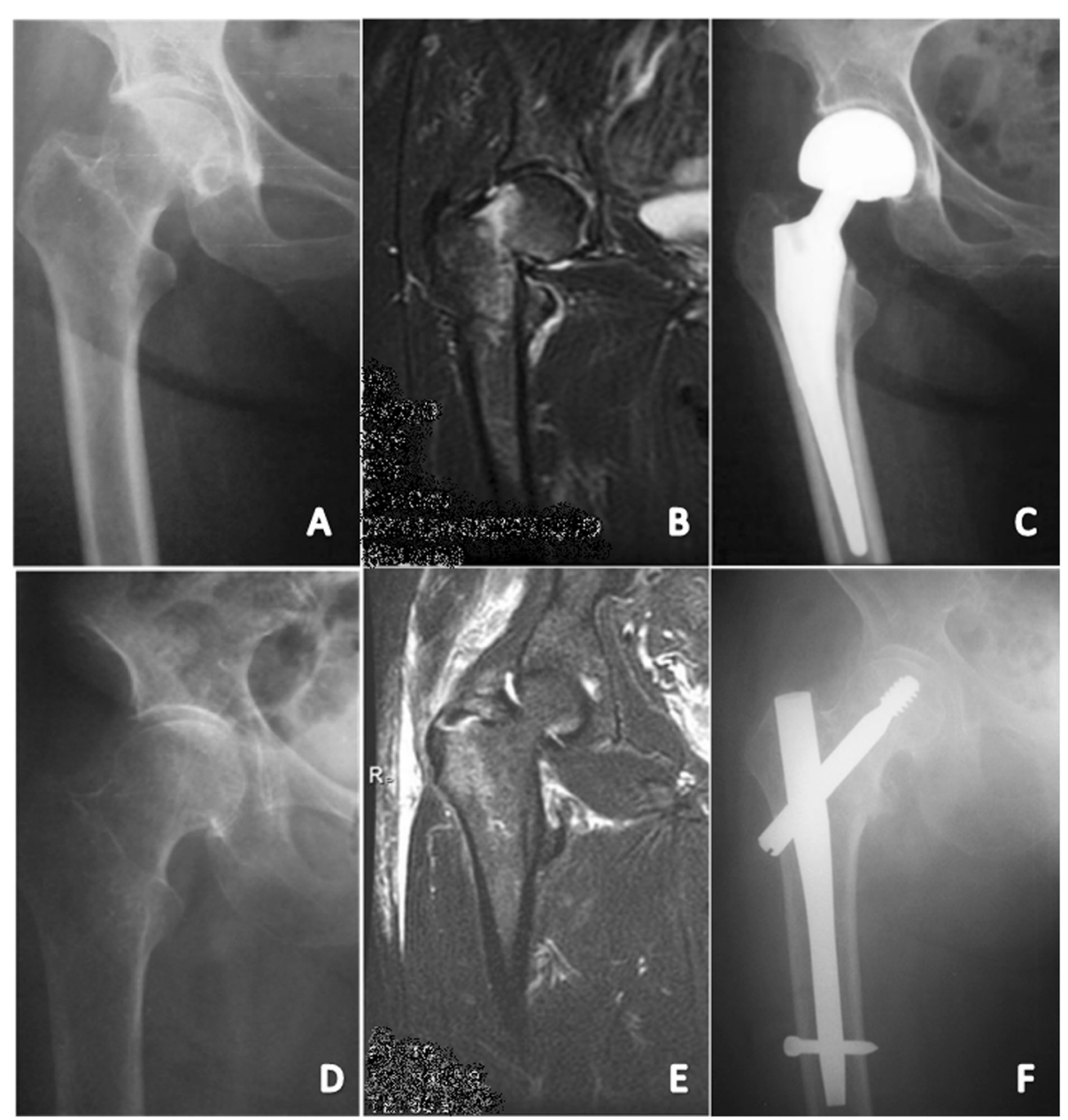

Fig. 3 a X-Ray of occult femoral neck fracture of the right hip. b MRI of the same case showing a Garden II femoral neck fracture. c Postoperative X-ray showing a uncemented bipolar hip. $\mathbf{d}$ X-Ray of occult pertrochanteric fracture of the right hip. e MRI of the same case showing a incomplete pertrochanteric fracture (>50\%). f Postoperative X-ray showing osteosynthesis with a Gamma 3 nail (Stryker, NJ)

\section{Discussion}

In the current study we present an imaging diagnosis protocol we have developed over the last 10 years using the best imaging modality available in an efficient manner and integrated this data with the selection of established treatment methods.

A hip MRI was preferred if available in the first $24 \mathrm{~h}$. For all the 47 cases investigated by a primary MRI a positive occult hip fracture diagnosis was obtained. An emergency CT scan was performed in 35 cases when the MRI was not available during the first $24 \mathrm{~h}$. This led to a true positive diagnosis in 12 cases. For the remaining 23 patients with negative $x$-rays and negative emergency $\mathrm{CT}$, but with persistent hip pain, the diagnosis was obtained next day by an MRI scan in 14 cases, or a CT scan in 9 cases $72 \mathrm{~h}$ later.

If clinical suspicion of a missed fracture arises with inconclusive radiological results [14] different imaging studies should be employed to investigate the primary cause of hip pain [3]. Further radiographic investigation of the hip is usually not carried out with standardized (oblique, Judet, inlet, outlet) [4] or experimental views [15] or particular digital image processing techniques [16].

Both orthopedic surgeons and radiologists alike consider MRI to be the gold standard in the detection of occult fractures [3, 6, 17-22],with $\mathrm{T}(1)$-weighted coronal MRI having $100 \%$ sensitivity while for $\mathrm{T}(2)$-weighted imaging there was $84.0 \%$ sensitivity [23]. Pandey et al. conducted an ER based study evaluating patients with traumatic hip pain and negative X-rays by MRI. They identified 22 fractures out of 33 suspected cases, but did not provide a total number of hip fractures for the period [21]. Dominguez et al. found $4.4 \%$ of patients with hip trauma and negative X-rays had a hip or pelvic fracture identified by MRI, representing $9.9 \%$ of the total number of hip fractures [4].

CT scanning has been employed for the detection of occult hip fractures [24] with $93 \%$ sensitivity and $95 \%$ specificity. A $72 \mathrm{~h}$ delay is advised for further improving 
sensitivity [25]. In a study by Dunker et al. of 193 hips in elderly patients with negative or inconclusive radiographs, CT scans performed within $24 \mathrm{~h}$ after low hip trauma detected 41 femoral neck and 68 pertrochanteric fractures [26]. Jordan et al. in a recent paper recognized the main advantage of CT in its availability (usually $24 / 7$ in a trauma center) and its smaller cost compared to MRI, in conformity with other authors, restating the role of CT in investigating occult hip fractures [2,5]. A comparative study by Lubovsky et al. concluded that MRI was more accurate and provided less misdiagnosis than $\mathrm{CT}$ on a relatively small group of 13 patients [19] while a paper by Hakkarinen reports 4 patients out of 24 occult hip fractures that had a negative 64 slice CT scan and a positive MRI, concluding that while CT scanning is a useful tool in detecting occult hip fractures (18 fractures out of 24 were identified by CT only) false negative results are possible even with 64 slice CT while no fractures were missed by MRI [11]. One of the main shortcomings of computer tomography is the emission of X-rays and the rather high dose of weight dependent ionizing radiation that the patient would receive that it is doubled if a repeat $72 \mathrm{~h} \mathrm{CT}$ is necessary [27].

Another imaging investigation described in literature is bone scintigraphy using technetium Tc $99 \mathrm{~m}$ polyphosphate, with a sensitivity of $98 \%$ [28]. It is dependent on the timing of the examination, with a lower sensitivity and specificity than MRI. It is also less reliable in very old patients and those with circulatory disturbances [12]. Furthermore, the time necessary for a MRI acquisition is much shorter, with trauma protocols taking less than fifteen minutes to perform [1]. Alternative modalities have been evaluated for their value in diagnosing proximal femur fractures. Sonography was found to have $100 \%$ sensibility and $65 \%$ specificity compared to MRI. It could therefore be proposed as a screening tool for occult hip fractures in the absence of readily available MRI [29]. Auscultatory percussion technique is another useful method to assess patients who present with posttraumatic hip pain and normal radiographs [30].

Limitations include the retrospective design of our study and the use of a 16 slice CT scanner, not the most efficient for diagnosing occult hip fractures [11]. The investigation was however performed by a senior radiologist and the evaluation was done together with a senior orthopedic surgeon limiting the bias and false negative results.

\section{Conclusions}

In conclusion, any patient with a suspected hip fracture (meaning a patient with significant posttraumatic hip pain, spontaneous, on palpation, mobilization or gait) and negative X-rays should receive further imaging exploration in the first $24 \mathrm{~h}$. If MRI is readily available it should be preferred as it has better accuracy in detecting occult hip fractures. If for whatever reason an MRI cannot be performed in the first $24 \mathrm{~h}$, an emergency CT scan should be used instead. This is often easier to accomplish, as CT is available round the clock in trauma centers and from our experience sufficient for establishing a diagnosis for the majority of patients. However in the case of a negative $24 \mathrm{~h} \mathrm{CT}$ the patient should be further investigated. Again the MRI should be the investigation of choice, and if not available followed by a repeat CT after $72 \mathrm{~h}$.

\section{Ethics approval and consent to participate}

The present study was brought before and approved by our institution IRB.

\section{Abbreviations}

AP: Antero-posterior; ER: Emergency room; CT: Computer tomography; ICD-10: International Statistical Classification of Diseases and Related Health Problems 10th Revision; MRI: Magnetic resonance imaging; X-ray: Radiography.

\section{Competing interests}

The authors declare that they have no competing interests.

\section{Authors' contributions}

Bogdan Deleanu is the main surgeon, having treated most of the cases involved in the study. He is also responsible for the main idea regarding the the conception and design of the study.

Radu Prejbeanu is treating surgeon for some of the cases involved. He also refined the concept of the study.

Dan Crisan is secondary surgeon and responsible for the design of the study, drafting the article, the acquisition, analysis and interpretation of data. Horia Haragus is secondary surgeon and analysis and interpretation of data and paper review.

Dinu Vermesan primary surgeon and critical reviewer.

Florin Birsasteanu was involved in the conception of the study improving it with important intellectual content.

Vlad Predescu provided critical review of the article.

Eleftherios Tsiridis provided valuable insights during the initial and ulterior review process and final approval of the version to be submitted.

All authors read and approved the final manuscript.

\section{Authors' information}

None.

\section{Acknowledgements}

\section{Funding}

No funding was received for the design, during the completion phase or for the publication of the manuscript.

\section{Author details}

${ }^{1}$ I-st Clinic of Orthopedics and Trauma, Pius Brinzeu Emergency Clinical County Hospital, 10 I. Bulbuca Blvd, 300737 Timisoara, Romania. ${ }^{2}$ Victor Babes University of Medicine and Pharmacy, 2 Eftimie Murgu Square, 300041 Timişoara, Romania. ${ }^{3}$ Department of Radiology, Pius Brinzeu Emergency Clinical County Hospital, 10 I. Bulbuca Blvd, 300737 Timisoara, Romania. ${ }^{4}$ St. Pantelimon Clinical Emergency Hospital, 340 - 342 Pantelimon Road, Sector 2, 033092 Bucharest, Romania. ${ }^{5}$ Carol Davila University of Medicine and Pharmacy, 8 Eroii Sanitari Blvd, 050474 Bucharest, Romania. ${ }^{6}$ Aristotle University Medical School, 54124 Thessaloniki, Greece.

Received: 26 September 2015 Accepted: 27 October 2015 Published online: 18 November 2015

\section{References}

1. Lim A. Occult hip fractures in elderly patients: is CT enough? General Medicine: Open Access. 2013;1(1):1-2.

2. Gill SK, Smith J, Fox R, Chesser TJS. Investigation of occult hip fractures: the use of CT and MRI. Scientific World Journal. 2013;2013:1-4. 
3. Cannon J, Silvestri S, Munro M. Imaging choices in occult hip fracture. J Emerg Med. 2009;37:144-52.

4. Dominguez S, Liu P, Roberts C, Mandell M, Richman PB. Prevalence of traumatic hip and pelvic fractures in patients with suspected hip fracture and negative initial standard radiographs-a study of emergency department patients. Acad Emerg Med. 2005;12:366-9.

5. Jordan RW, Dickenson E, Baraza N, Srinivasan K. Who is more accurate in the diagnosis of neck of femur fractures, radiologists or orthopaedic trainees? Skeletal Radiol. 2012;42:173-6.

6. National Clinical Guideline Centre (UK). The Management of Hip Fracture in Adults. London: Royal College of Physicians (UK); 2011.

7. Lee K-H, Kim H-M, Kim Y-S, Jeong C, Moon C-W, Lee S-U, et al. Isolated fractures of the greater trochanter with occult intertrochanteric extension. Arch Orthop Trauma Surg. 2010;130:1275-80.

8. O'Grady SD. Mosby, 2009. Mosby's dictionary of medicine, nursing \& health professions. Mosby Elsevier. Nurse Educ Pract. 2010;10:0. ISBN:978-0-323-04937-5.

9. Lakshmanan P, Sharma A, Lyons K, Peehal JP. Are occult fractures of the hip and pelvic ring mutually exclusive? J Bone Joint Surg Br. 2007;89:1344-6.

10. Agency for Healthcare Research and Quality [http://www.guideline.gov/ content.aspx?id=47668].

11. Hakkarinen DK, Banh KV, Hendey GW. Magnetic resonance imaging identifies occult hip fractures missed by 64-slice computed tomography. J Emerg Med. 2012:43:303-7.

12. Rubin G, Malka I, Rozen N. Should we operate on occult hip fractures? Isr Med Assoc J. 2010;12:316-7.

13. Rizzo PF, Gould ES, Lyden JP, Asnis SE. Diagnosis of occult fractures about the hip. Magnetic resonance imaging compared with bone-scanning. J Bone Joint Surg Am. 1993;75:395-401.

14. Helland EB, Tollefsen I, Reksten G. Radiographic diagnosis of the occult hip fracture: experience in 16 patients. Acta Orthop. 2000;71:639-41.

15. Harding J, Chesser TJS, Bradley M. The bristol hip view: its role in the diagnosis and surgical planning and occult fracture diagnosis for proximal femoral fractures. Scientific World Journal. 2013;2013:703783-4.

16. Botser IB, Herman A, Nathaniel R, Rappaport D, Chechik A. Digital image enhancement improves diagnosis of nondisplaced proximal femur fractures. Clin Orthop Relat Res. 2009:467:246-53.

17. Frihagen F, Nordsletten L, Tariq R, Madsen JE. MRI diagnosis of occult hip fractures. Acta Orthop. 2005;76:524-30.

18. Hossain M, Barwick C, Sinha AK, Andrew JG. Is magnetic resonance imaging (MRI) necessary to exclude occult hip fracture? Injury. 2007;38:1204-8.

19. Lubovsky O, Liebergall M, Mattan Y, Weil Y, Mosheiff R. Early diagnosis of occult hip fractures MRI versus CT scan. Injury. 2005;36:788-92.

20. Nachtrab O, Cassar-Pullicino VN, Lalam R, Tins B, Tyrrell PNM, Singh J. Role of MRI in hip fractures, including stress fractures, occult fractures, avulsion fractures. Eur J Radiol. 2012;81:3813-23.

21. Grad WB, Desy NM. Bilateral occult hip fracture. CJEM. 2012;14(6):372-7.

22. Lee YP, Griffith JF, Antonio GE, Tang N, Leung KS. Early magnetic resonance imaging of radiographically occult osteoporotic fractures of the femoral neck. Hong Kong Med J. 2004;10:271-5.

23. Imata T, Nozawa S, Dohjima T, Yamamoto T, Ishimaru D, Tsugita M, et al, The value of T1-weighted coronal MRI scans in diagnosing occult fracture of the hip. J Bone Joint Surg Br. 2012;94:969-73.

24. Collin D, Dunker D, Göthlin JH, Geijer M. Observer variation for radiography, computed tomography, and magnetic resonance imaging of occult hip fractures. Acta Radiol. 2011;52:871-4.

25. Gangopadhyay S, Akra GA, Nanu AM. Occult hip fractures in the elderly: a protocol for management. Eur J Orthop Surg Traumatol. 2006;17:153-6.

26. Dunker D, Collin D, Göthlin JH, Geijer M. High clinical utility of computed tomography compared to radiography in elderly patients with occult hip fracture after low-energy trauma. Emerg Radiol. 2012;19:135-9.

27. Israel GM, Cicchiello L, Brink J, Huda W. Patient size and radiation exposure in thoracic, pelvic, and abdominal CT examinations performed with automatic exposure control. AJR Am J Roentgenol. 2010;195:1342-6.

28. Brunner LC, Eshilian-Oates L, Kuo TY. Hip fractures in adults. Am Fam Physician. 2003;67:537-42.

29. Safran O, Goldman V, Applbaum Y, Milgrom C, Bloom R, Peyser A, et al. Posttraumatic painful hip: sonography as a screening test for occult hip fractures. J Ultrasound Med. 2009;28:1447-52.

30. File P, Wood JP, Kreplick LW. Diagnosis of hip fracture by the auscultatory percussion technique. Am J Emerg Med. 1998;16:173-6.

\section{Submit your next manuscript to BioMed Central and take full advantage of:}

- Convenient online submission

- Thorough peer review

- No space constraints or color figure charges

- Immediate publication on acceptance

- Inclusion in PubMed, CAS, Scopus and Google Scholar

- Research which is freely available for redistribution

Submit your manuscript at www.biomedcentral.com/submit 